\title{
THE NOVEL MESOPOROUS SILICA AEROGEL MODIFIED WITH PROTIC IONIC LIQUID FOR LIPASE IMMOBILIZATION
}

\author{
Anderson S. Barbosa ${ }^{a, c}$, Jessica A. Lisboa ${ }^{a}$, Matheus A. O. Silva ${ }^{a}$, Nayára B. Carvalho ${ }^{a}$, Matheus M. Pereirab, Alini T. \\ Fricks $^{\mathrm{a}}$, Silvana Mattedid ${ }^{\mathrm{d}}$ Álvaro S. Lima ${ }^{\mathrm{a}}$, Elton Franceschic ${ }^{\mathrm{c}}$ and Cleide M. F. Soares ${ }^{\mathrm{a}, *}$ \\ anstituto de Tecnologia e Pesquisa, Universidade Tiradentes, Avenida Murilo Dantas 300, 49032-490 Aracaju - SE, Brasil \\ 'Departamento de Química, Universidade de Aveiro, Campus de Santiago, 3810-193, Aveiro, Portugal \\ ${ }^{\mathrm{c}}$ Núcleo de Estudos em Sistemas Coloidais, Universidade Tiradentes, Avenida Murilo Dantas 300, 49032-490 Aracaju - SE, Brasil \\ dDepartamento de Química, Universidade Federal da Bahia, Rua Barão de Geremoabo 147, Campus Ondina, 40170-115 Salvador \\ - BA, Brasil
}

Recebido em 24/08/2015; aceito em 22/12/2015; publicado na web em 11/02/2016

\begin{abstract}
Mesoporous silica supports (aerogels) were used to immobilize Burkholderia cepacia lipase (BC) by encapsulation (EN or ENIL), physical adsorption (ADS or ADSIL) and covalent binding (CB or CBIL) into or onto the aerogel modified with protic ionic liquid (PIL). Yield immobilization (Ya) and operational stability were determined by the hydrolytic reaction of olive oil. Ya (37\% to $83 \%$ by physical adsorption) and operational stability ( 2 to 23 batches by encapsulation) increased when the support was modified with PIL. For immobilized derivates observed by the BET method, in this case ADS and CB for ADSIL and CBIL, increased pores size was observed, possibly due to the higher amount of BC immobilized conferring Ya and operational stability. This effect was probably attributed to the entry of the enzyme into the pores of the silica aerogel structure. SEM images showed a change in the structure and properties of immobilized lipase derived with PIL. A characteristic FTIR band was obtained for the silanol groups and amides I, IV and V, demonstrating the efficiency of immobilization of BC. The most efficient biocatalysts were ADSIL with regard to yield immobilization and ENIL for operational stability.
\end{abstract}

Keywords: aerogel; lipase; immobilization; protic ionic liquid.

\section{INTRODUCTION}

The Aerogels and xerogels are formed via hydrolysis and polycondensation reactions of silica precursors, such as tetraethylorthosilicate (TEOS), and care must be taken not to cause collapse or reduction in surface area and pore size. Some techniques have been developed for solvent extraction. The technique for the synthesis of aerogels occurs in supercritical conditions and was created by Kistler, ${ }^{1}$ with the goal of producing a dry gel with superior physical properties and the same volume of moist gel as other methods, replacing the solvent in gas pores. ${ }^{2}$ Aerogels based on silica and other metal oxides are extremely porous, with high specific surface areas and low density. Studies have shown the use of aerogels for lipase encapsulation by the sol-gel technique, as the enzyme is physically encapsulated in a rigid glass framework that permits stabilisation of the enzymes and gives a tertiary structure because of the tight gel network..$^{3-5}$

There are various methods for enzyme immobilization which may be physical or chemical. Among them are physical adsorption, covalent binding, crosslinking, encapsulation, and others. ${ }^{6,7}$ The biocatalysts immobilized by encapsulation in aerogels obtained by the sol-gel technique and drying pressurised medium have been studied for different applications such as transesterification, ${ }^{3,8,9}$ esterification, ${ }^{10}$ and other reactions. Little literature reports immobilization by physical adsorption and covalent binding with aerogels. Another way to increase the activity and stability of immobilized enzymes is the use of additives to stabilise enzymes within sol-gel matrices.

The study of different additives in the literature is limited to xerogels, such as Aliquat 336, in the immobilization of Bacillus sp. ITP-001 showed higher yields in hydrolysis reaction (71\% and 40\%, respectively) than the same immobilized lipase without additives. ${ }^{11}$ The application of ionic liquids (IL) as additive during the process of synthesising supports and immobilization of biocatalysts are also recent areas of research regarding xerogels, ${ }^{12-15}$ as regards aerogels some studies using IL in the matrix with drying pressurized was found, but for biocatalysts immobilized in aerogel matrices in the presence the protic ionic liquid yet not is found.

The potential of ionic liquids has led to their use as additives in the process of sol-gel immobilization, for the purpose of protecting against inactivation of enzymes because of the release of alcohol and shrinking of the gel during the immobilization process and this positive was confirmed effect using xerogel. ${ }^{12}$ The ionic liquids obtained from amines, and organic and inorganic acids, the so-called protic ionic liquids (PIL), are characterised by low cost and simplicity of synthesis, and are considered as good alternative solvents with potential applications in industry, including those involving biocatalysis. ${ }^{16-18}$ Aerogels have been used as organic solvents in many biocatalytic reactions to improve enzymatic activity and stability ${ }^{9}$ and yet the use of protic ionic liquids in immobilization methods with aerogels is unknown.

The novel mesoporous silica aerogel modified with protic ionic liquid for lipase immobilization onto silica aerogel yet was not observed in other studies and unique described in the literature was available the immobilization of lipase by encapsulation into silica aerogel, ${ }^{19}$ and yet are necessary new studies this process.

In this context, the present study has focused on the use of different immobilization methods onto silica aerogel in the presence of PIL to evaluate the activity and operational stability of immobilized biocatalysts.

*e-mail: cleide.soares@pq.cnpq.br 


\section{EXPERIMENTAL}

\section{Materials}

The lipase (E.C.3.1.1.3) used in the present study was from Burkholderia cepacia (Amano Lipase) and was purchased from Sigma Aldrich (Japan). Aerogels were fabricated with silane precursor tetraethoxysilane (TEOS, 98\%) supplied by Across Organic (NJ, USA), used without further purification, and the protic ionic liquid N-methylmonoethanolamine pentanoate $\left(\mathrm{C}_{5}\right)$ (Figura 1) was supplied by the Federal University of Bahia (UFBA, Brazil). Ethanol (minimum $99 \%$ pure), ammonia (minimum $28 \%$ pure), hydrochloric acid (minimum $36 \%$ pure) and gum arabic were obtained from Synth (São Paulo, Brazil). Silane $\gamma$-aminopropyltriethoxysilane ( $\gamma$-APTS) and glutaraldehyde ( $25 \%$ solution) were from Sigma. All other chemicals were of analytical grade from various suppliers.

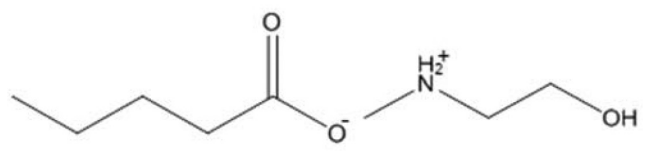

Figura 1. Structure of protic ionic liquid (PIL) (N-methylmonoethanolamine pentanoate)

\section{Aerogel silica supports synthesis}

A methodology previously established by Souza and colleagues ${ }^{12}$ was used with some modifications, and is briefly described as follows: $30 \mathrm{~mL}$ of TEOS was dissolved in $36 \mathrm{~mL}$ of absolute ethanol under an inert nitrogen atmosphere. To this was added $0.22 \mathrm{~mL}$ of hydrochloric acid dissolved in $5 \mathrm{~mL}$ of slowly added ultra-pure water, and the mixture was agitated $(200 \mathrm{rpm})$ for $90 \mathrm{~min}$ at $35^{\circ} \mathrm{C}$. In case the presence of additives, $1 \%(\mathrm{w} / \mathrm{v})$ of the protic ionic liquid $\mathrm{C}_{5}$ was simultaneously and separately added. Afterwards, $1.0 \mathrm{~mL}$ of ammonium hydroxide dissolved in $6.0 \mathrm{~mL}$ of ethanol (hydrolysis solution) was added to the sol-gel reaction, and the mixture was kept under static conditions for $24 \mathrm{~h}$ to complete polycondensation. The bulk gel was then washed with heptane and acetone. The material was collected and dried using $\mathrm{CO}_{2}$ pressurized for $6 \mathrm{~h}$ in an extractor with the temperature and the pressure kept constant at $25^{\circ} \mathrm{C}$ and 100 bar, respectively, which leads to the formation of aerogels according to Barbosa and colleagues. ${ }^{19}$ For comparison purposes pure silica aerogel was prepared in the absence (SA) or presence (SAIL) of additives. The mesoporous silica aerogel obtained was used for immobilising commercial BC by physical adsorption (ADS) and covalent binding (CB).

\section{Aerogel silica immobilization}

The immobilization process of the enzymes into or onto mesoporous silica supports (aerogel) was carried out by three methods in the absence or presence of $\mathrm{C}_{5}$, respectively: encapsulation (EN or ENIL) in other article, ${ }^{19}$ and this work was tested lipase immobilization by physical adsorption (ADS or ADSIL) and covalent binding (CB or CBIL). For the preparation of lipase-encapsulated derivatives, a procedure was adopted involving the addition of the lipase from B. cepacia $(870.71 \mathrm{U})$ in the absence and presence of additives $(1 \%$ (w/v) of the protic ionic liquid $\mathrm{C}_{5}$ ). ${ }^{12}$ The drying of gel was in the same conditions of the item above.

Lipase was immobilized by ADS on the aerogel silica support following the methodology of Soares and colleagues ${ }^{4}$ with some modifications. For the covalent binding the aerogel silica support was silanised with $\gamma$-APTS followed by reaction with glutaraldehyde solution, and then lipase was immobilized by $\mathrm{CB}$ according to the procedure described by Soares and colleagues. ${ }^{4}$ The enzyme support (CB) and enzyme support additive (CBIL) were used for immobilization in accordance with Soares and colleagues. ${ }^{4}$

\section{Enzymatic activity}

Enzymatic activities of both free and immobilized lipase samples were assayed by the olive oil hydrolysis method according to a modification used by Soares and colleagues. ${ }^{4}$ One unit $(U)$ of enzyme activity was defined as the amount of enzyme that liberated $1 \mu \mathrm{mol}$ of free fatty acid per min $\left(\mu \mathrm{mol} \mathrm{min}{ }^{-1}\right)$ under assay conditions $\left(37^{\circ} \mathrm{C}\right.$, $\mathrm{pH} 7.0,80 \mathrm{rpm}$ ). Analyses of the hydrolytic activities of biocatalysts immobilized were used to determine the yield $\eta(\%)$ according to Equation 1

$$
\eta(\%)=\frac{U_{s}}{U_{o}} \times 100
$$

in which $U_{s}$ is the total enzyme activity recovered on the support and $U_{o}$ is the enzyme unit offered for immobilization.

\section{Reusability of immobilized lipase}

The operational stability of the immobilized system was determined in hydrolysis reactions in consecutive batches with reuse of immobilized lipase from Burkholderia cepacia in silica aerogel. This study was employed in all batches, using the same mass of immobilized biocatalyst. Were carried out batches $30 \mathrm{~min}$ at temperature of $37^{\circ} \mathrm{C}$ and $\mathrm{pH}$ 7.0. The immobilized enzyme was then washed with hexane once and used for the next cycle of hydrolysis. This procedure was repeated for several cycles.

\section{Sample characterization}

To characterise the porous texture and the network structure of the silica aerogel and immobilized lipase derivatives were calculated with the Brunauer-Emmett-Teller method..$^{20}$ Pore volume and average pore diameter was calculated based on the model developed by Barret, Joyner and Halenda (BJH) for mesoporous samples and for microporous samples by the $t$-method. Surface areas were evaluated according to their $\mathrm{N}_{2}$ adsorption at $77 \mathrm{~K}$ with BET apparatus software (NOVA 1200e - Surface Area \& Pore Size Analyser, Quantschrome Instruments - version 11.0). Prior to analysis, samples were subjected to thermal treatment at $120^{\circ} \mathrm{C}$ for $48 \mathrm{~h}$, to eliminate any water existing within the pores of the solids.

Thermogravimetric (TG) curves were obtained with Shimadzu DTG-60H simultaneous DTA-TG apparatus, under a nitrogen atmosphere that started at room temperature and went up to $1000{ }^{\circ} \mathrm{C}$, increasing at a heating rate of $20^{\circ} \mathrm{C} \mathrm{min}^{-1}$. Scanning electron microscopy (SEM; model Hitachi SU-70) was also used to characterise the surfaces of the samples. The samples of immobilized lipase in the presence and absence of additive were subjected to FTIR analysis (spectrophotometer FTIR BOMEMMB-100). Spectra were obtained in the wavelength range from 400 to $4000 \mathrm{~cm}^{-1}$.

\section{RESULTS AND DISCUSSION}

\section{Screening of immobilization method}

\section{Influence of immobilization method in silica aerogel in the enzymatic activity}

The screening of immobilization methods is required to confirm the positive or negative effect of ionic liquid on the synthesis of 
immobilized biocatalysts using drying pressurised. Three immobilization procedures in the absence and presence of protic ionic liquid were realized in this work. In the first, the enzyme was immobilized by physical adsorption; in the second, the lipase was immobilized by covalent binding on the silica aerogels previously functionalised with $\gamma$-APTS and activated with glutaraldehyde and, in the third, the enzyme was encapsulated.

The behaviour of the lipase immobilised by the sol-gel technique depends on the physical structure of the support, the chemical and physical proprieties of the lipase and the drying method employed. ${ }^{21}$ For lipase encapsulation into the silica aerogel, was determined $45 \%$ of total activity recovery yield using ionic liquid (ENIL) and $37 \%$ in the absence of PIL (EN) in according described by research group in article published this periodic (Figure 2).$^{19}$ In this work, was verified the enzyme immobilization onto surface of the aerogel utilizing physical adsorption or covalent binding. For BC lipase immobilized by physical adsorption in silica aerogel, we verified an increase in the total activity recovery yield of 70.31 (ADS) to $83 \%$ when silica aerogel with PIL was used (ADSIL). For BC lipase immobilization by covalent binding, the results showed that aerogel supports in the presence of the additive achieved a total activity recovery yield of $69.92 \%$ (CBIL), lower than the recovery exhibited in physical adsorption $(82.95 \%)$. The catalytic efficiency of the enzyme was 1.8 times higher as regards the total activity recovery yield than immobilized biocatalyst in the absence of PIL.

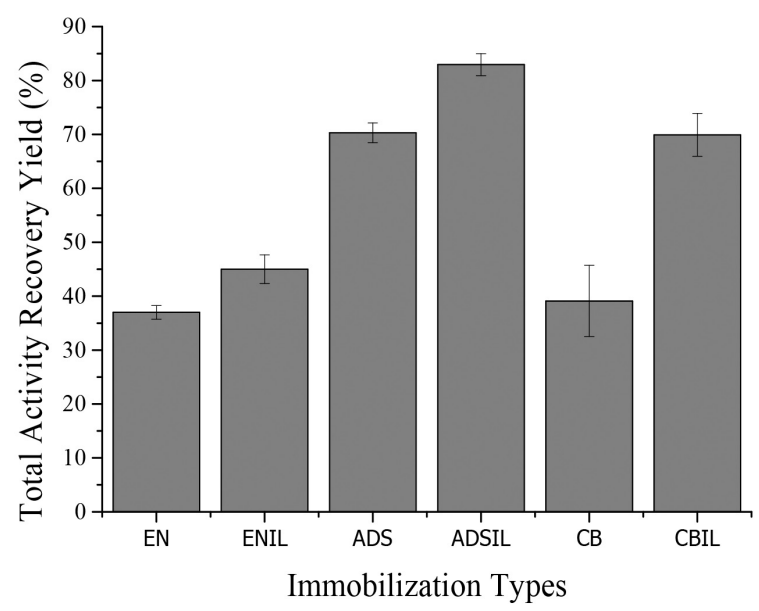

Figure 2. The total activity recovery yield ( $\eta \%)$ results for the immobilization of lipase on silica aerogels with increase from $37 \%$ to $83 \%$, for encapsulation (EN or ENIL), ${ }^{19}$ physical adsorption (ADS or ADSIL) and covalent binding (CB or $C B I L)$

This great variability sometimes depends on the use of PIL in the immobilizations tested. The LI in silica aerogels, which have a hydrophobic character, permits the formation of a hydration layer around the enzyme promoting enzymatic activity and pushing the water closer to the enzyme. The literature reports that the commercial lipase solution from Candida antarctica, Lipozyme ${ }^{\circ}$, encapsulated in silica aerogels reinforced with silica quartz fibre felt in the presence of LIs (1-butyl-3-methylimidazolium tetrafluoroborate, $\left[\mathrm{C}_{4}\right.$ min $] \mathrm{BF}_{4}$ and 1-butyl-4-methylpyrimidic tetrafluoroborate, $\left.\left[\mathrm{C}_{4} \mathrm{pyr}\right] \mathrm{BF}_{4}\right)$ showed $90 \%$ conversion to biodiesel in $50 \mathrm{~h}$ of reaction at $40{ }^{\circ} \mathrm{C} .{ }^{9}$ For xerogels, Zarcula and colleague ${ }^{22}$ obtained positive effects using an ionic aprotic liquid of a more hydrophobic character (1-octyl-3-methylimidazolium tetrafluoroborate, [C8mim] [BF4]) in the immobilization of the sol-gel technique lipase from Pseudomonas fluorescens. Moreover, works developed by the group ${ }^{12,16}$ showed that the use of ionic protic liquids has various effects on and causes structural change in the support.
The use of the PILs of N-methylmonoethanolamine family appeared to be more efficient in BC lipase immobilization into support with the traditional drying method. According to the literature on the subject the use of an additive can coat the surface of silica and protect the channels and the enzyme during shrinkage and collapse of the gel pores as well as acting as a stabiliser to protect the enzyme from inactivation in immobilization by encapsulation and the release of alcohol during hydrolysis of a silicon alkoxide. ${ }^{23,24}$ The presence of PIL can be actuated as a polyvinylalcohol (PVA) additive during the immobilization or synthesis of the support, possibly by modifying the hydrophobicity of the microenvironment because of its influence on the moisture of lipase..$^{25}$

The lipase immobilization on aerogels supports by physical adsorption, the literature reports inferior results to those obtained when immobilized lipase from Candida rugosa on methyl-modified silica aerogel, which reached the total activity recovery yield was $56.44 \%$ in hydrolysis reaction. ${ }^{26}$ The literature thus suggests that the use of immobilized BC lipase in the absence and presence of PIL in aerogel supports showed promising performance in the hydrolysis of olive oil. Comparing the different methods of immobilization in the presence and absence of PIL we observed significant influence of the type of immobilization and presence of PIL on enzymatic activity, confirming reports in the literature. ${ }^{12}$ However, there are no studies about support utilized for physical adsorption and covalent binding in the presence of PIL, except for silica aerogel.

To justify the increase of the total activity recovery yield we analysed operational stability beyond the physico-chemical and morphological properties of the aerogel support and immobilized biocatalysts. For adequate selection of the immobilization method, operational stability is an important factor in terms of its use in industrial processes.

\section{Operational stability of the immobilized biocatalysts}

Operational stability is based on the operational half-life, i.e. the operation time needed to reduce its original activity to $50 \%$. This study showed the necessity for evaluation of the operational stability of biocatalysts immobilized on or into the surface of mesoporous silica supports (EN, ENIL, ADS, ADSIL, CB and CBIL) because of weak Van der Waals interaction, hydrogen bonding interaction and electrostatic interaction between the supports in the presence or absence of the addictive and enzyme. ${ }^{27}$

The operational stability of biocatalysts immobilized by encapsulation (EN and ENIL) in the reaction of hydrolysis of olive oil, was published in article of the research group ${ }^{19}$ and can be seen in Figure 3 (a) as a slow reduction of the relative activity in the two immobilized systems. In biocatalysts encapsulated (EN) half-life was 6.5 hours, totalling 13 reuses. For ENIL the value of relative activity was lower compared to EN, but in the 11th reuse this value was higher than the biocatalyst without the additive and remained stable for 23 cycles above $70 \%$, and the half-life was almost double (11.4 hours). The systems of silica aerogels immobilized by physical adsorption (ADS and ADSIL) showed a loss of enzymatic activity in $50 \%$ for the hydrolysis reaction in cycle number 2 , with a half-life of 0.73 hours for ADSIL and 0.88 hours for ADS, proving them unviable for industrial applications (Figure $3 \mathrm{~b}$ ). The biocatalysts immobilized by covalent binding on silica aerogel showed an increase in the number of reuses compared to immobilization by physical adsorption. In the absence of PIL (CB) there was a total of seven reuses and a half-life of 2.8 hours and in the presence of PIL (CBIL) 15 reuses whereby residual activity was maintained by approximately seven cycles with $120 \%$ of relative activity.

The operational stability of the BC lipase encapsulated in silica aerogel in the presence of PIL showed more stable, with $70 \%$ of its 
relative activity after the 20th reuse. The enzyme immobilized by encapsulation into silica aerogel prevents enzyme release during washing steps employed in the recycling reaction because it provides a cage around the immobilized enzyme, preventing the leaching of the enzyme from the support and resulting in greater reusability of the biocatalyst. ${ }^{28}$ The loss of activity during reuse of the biocatalyst immobilized by physical adsorption can be explained by electrostatic interactions or weak links in the support/enzyme, which resulted in desorption during the washing or other process stages. ${ }^{29}$ Reuse of Rizophus oryzae lipase (ROL) immobilized by adsorption on calcium carbonate and oxidised cellulose carried out by producing oleate butyl were obtained by six and three cycles respectively in the work of Guamgui et al., ${ }^{30}$ and Karra-Châabouni et al., ${ }^{31}$

The CB and CBIL showed an increase in the number of reuses compared with ADS and ADSIL. This behaviour occurred because immobilization by covalent binding becomes stronger binding of enzymes on the silica aerogel to prevent leaching because of the chemical bonding between the amino groups of the lipase and the terminal aldehyde of the support is irreversible and resist. ${ }^{32,33}$ It is worth mentioning that some reports demonstrate that covalent binding of the enzyme with a suitable bifunctional agent improves the stability of the immobilized enzymes. In the immobilization of lipases from porcine pancreas by the method of covalent binding in the presence of PVA as additive in silica aerogel, there was a half-life of 6 hours at 10 cycles of reuse with residual activity of $70 \%$ in the final reuse in the emulsification reaction of olive oil. ${ }^{34}$

For the biocatalysts EN, ENIL and CBIL we observed an increase in the recovery activity in the second reuse compared with the first. This result can be explained by the removal of impurity, protein residues or even smaller proteins the enzyme present in the enzyme solution for the gel during successive reuse reactions. ${ }^{3,9-10}$ This removal can be seen as a dialysis direct enzyme solution during the process of encapsulation in the gel and the phenomenon can disrupt the connection of the gel pores and thus limit the increase in diffusion of substrate. ${ }^{3}$ In The literature shows that immobilized BC lipase in mesoporous silica supports can cause increase in the second reuse of $75 \%$ compared with the first, and that the catalytic activity of enzyme decreases to approximately $60 \%$ after the 11 th reuse. ${ }^{3,10}$ It is important to remember that there are no studies yet was realized about the influence of the protic ionic liquid in silica aerogels.

\section{Morphological and physic-chemical characterization}

The characterization of the novel mesoporous silica aerogel modified with protic ionic liquid for lipase immobilization into or onto support yet was not verified in the literature, in this work was studied physical-chemical and morphological characteristic.

\section{Specific surface area and porous properties}

The characterization of the porosity of silica aerogel, whether pure or containing immobilized biocatalysts, is an important factor because it helps our understanding of the results of enzymatic activity. Methods based on gas adsorption are the most convenient for the study of the porous properties of solid materials using volumetric measurements of the adsorbed gas quantities. The specific surface area, pore and volume size of the immobilized biocatalysts and silica aerogel in the absence and presence of PIL and the addition of PILs changed the morphological structure of the immobilized biocatalysts, as summarized in Table 1 . The largest pore size and surface area were obtained when the protic ionic liquid was increased in the sol-gel process. Surface area and mean pore diameter results indicated a significant influence of protic ionic liquid on the derivatives properties.
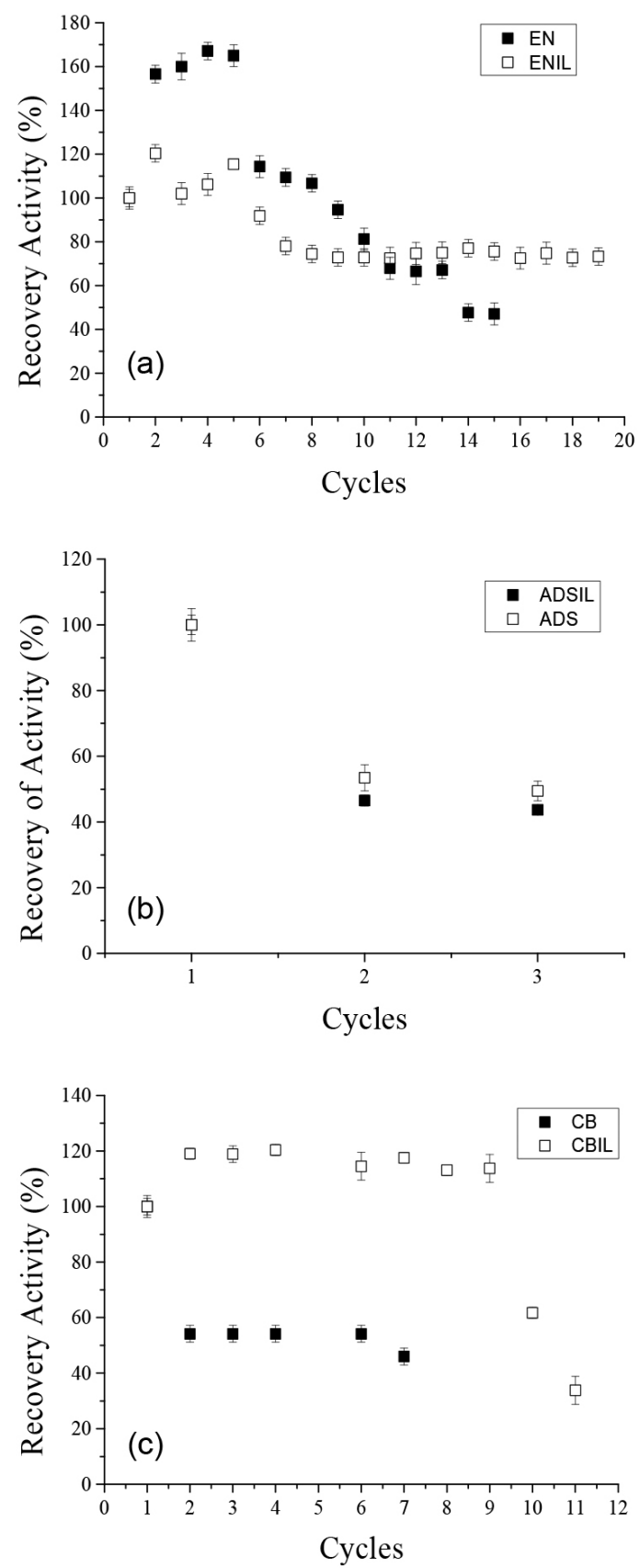

Figure 3. Recovery activity of lipase immobilized by: (a) encapsulation, ${ }^{19}(b)$ physical adsorption and (c) covalent binding in the presence and absence of ionic liquids in the silica aerogel

Table 1. Influence of protic ionic liquid used as an additive in silica aerogel immobilized lipase on the textural properties of adsorption-desorption of nitrogen

\begin{tabular}{cccc}
\hline Sample & $\begin{array}{c}\text { Surface area } \\
\left(\mathrm{m}^{2} \mathrm{~g}^{-1}\right)\end{array}$ & $\begin{array}{c}\text { aPore volume } \\
\left(\mathrm{cm}^{3} \mathrm{~g}^{-1}\right)\end{array}$ & $\begin{array}{c}\text { Pore diameter } \\
(\mathrm{nm})\end{array}$ \\
\hline SA & 81 & 0.04 & 1.1 \\
SAIL & 322 & 0.8 & 5.0 \\
EN & 131 & 0.5 & 8.2 \\
ENIL & 147 & 0.4 & 5.9 \\
ADS & 248 & 0.3 & 2.4 \\
ADSIL & 289 & 0.6 & 4.7 \\
CB & 272 & 0.2 & 1.7 \\
CBIL & 235 & 0.6 & 5.6 \\
\hline
\end{tabular}

apore volume calculated from nitrogen desorption. 
The SA with protic ionic liquid showed a type IV isotherm with a hysteresis loop (Figure 4a), which are typically exhibited by mesoporous solids. ${ }^{35}$ The results observed for SAIL in the $\mathrm{N}_{2}$ adsorption-desorption tests concur with those of the isotherms with $\mathrm{H} 1$ hysteresis loops, which is a characteristic of mesoporous materials and usually associated with pores with narrow necks and wide bodies

(a)

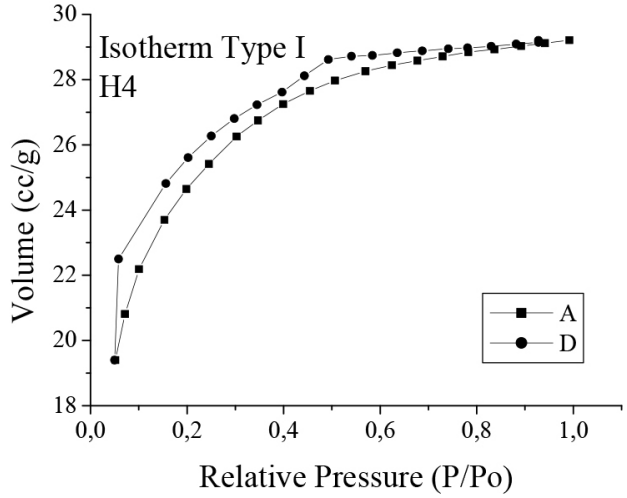

(c)

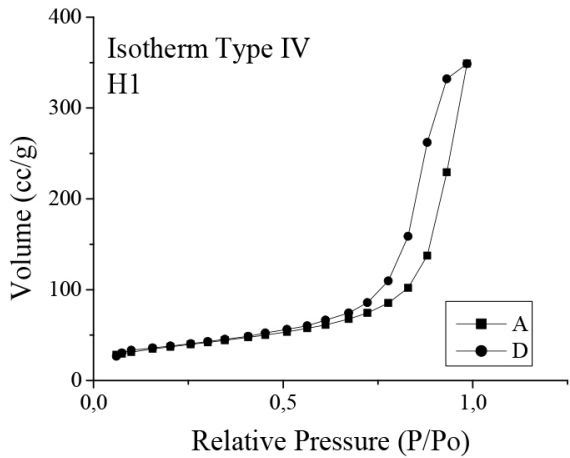

(e)

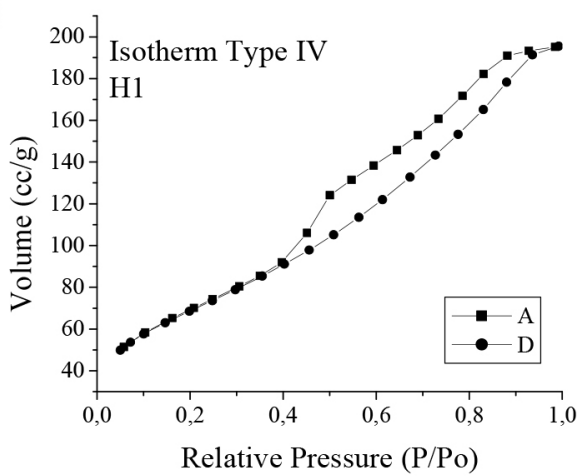

(g)

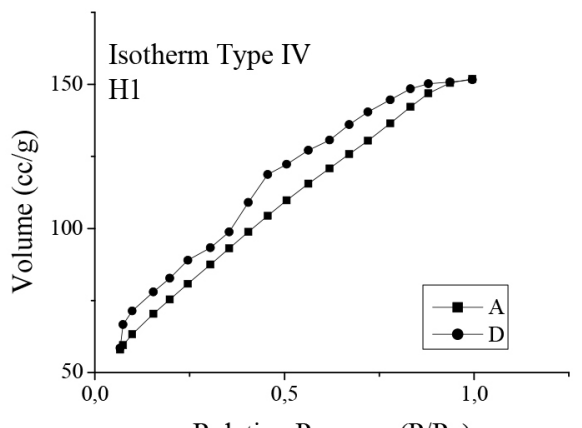

(Figure 4b). Similar results were obtained for the immobilized lipase samples, which all showed a type IV isotherm with hysteresis loop H1.

The characterization of the porosity of silica aerogel when compared with pure silica aerogel (SA), the control, the support prepared in the presence of PIL only (without enzyme, SAIL) showed increased surface area and similar values for pore diameter and pore volume

(b)

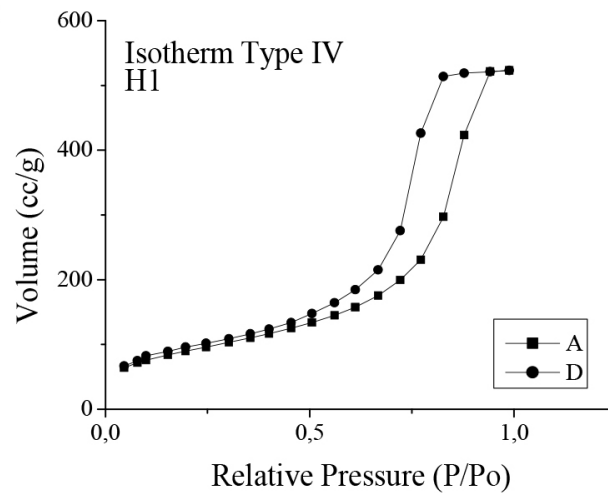

(d)

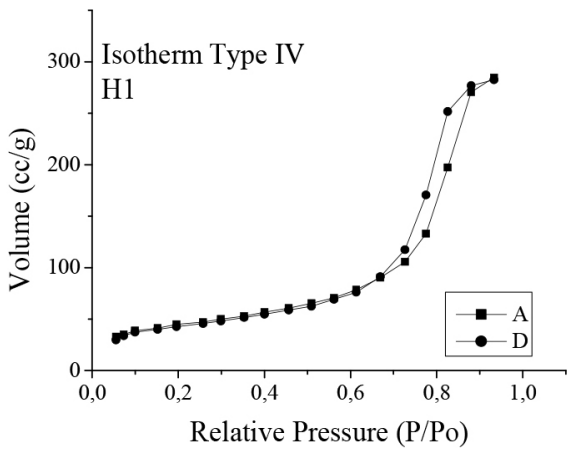

(f)

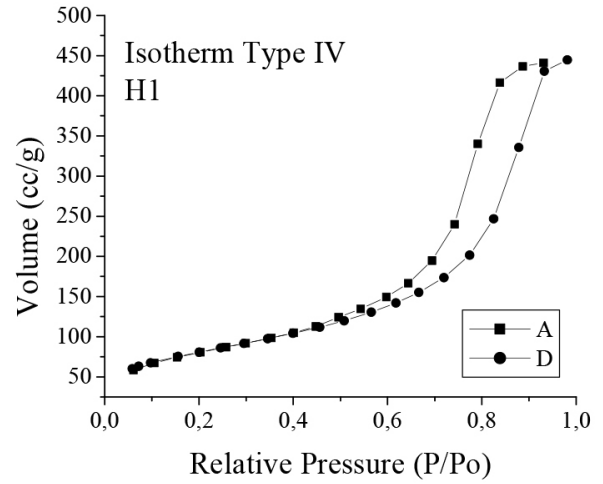

(h)

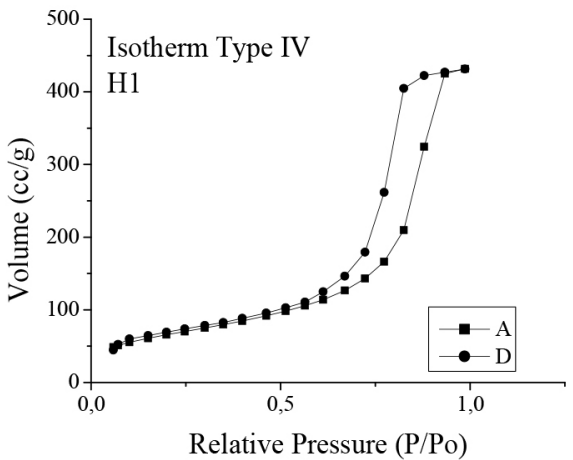

Figure 4. Nitrogen adsorption-desorption isotherms of pure silica aerogel and immobilized samples: $(a) S A,(b) S A I L,(c) E N,(d) E N I L,(e) A D S,(f) A D S I L,(g)$ $C B$ e (h) CBIL 
(Table 1), suggesting that the use of PIL caused structural variations in the silica aerogel control. Hence, from the nitrogen adsorption-desorption measurements it is clear that the ionic liquid acted as a pore-forming agent. This profile was similar to that observed by Vila-Real and colleagues, ${ }^{36}$ who reported that the addition of ionic liquids in the sol-gel immobilization process plays an important role in enzyme performance by affecting the structural characteristics of the immobilized biocatalyst. The average pore size and ionic liquid content in the gel have a direct relation with each other and hence the presence of IL inside the gel increases the pore radius to a higher value. ${ }^{35}$ On the other hand, the results show that the addition of protic ionic liquids decreased the specific surface area for ENIL in relation to silica aerogel with IL (SAIL), in agreement with results reported by Souza and colleagues ${ }^{12}$ for a BC commercial lipase and PILs. This is possibly because of the partial adsorption of the enzyme on the external surface of the hydrophobic matrix. ${ }^{11}$

The biocatalyst immobilized by encapsulation in the presence of PIL (ENIL) had positive effects on the surface area $\left(147 \mathrm{~m}^{2} \mathrm{~g}^{-1}\right)$ compared with the biocatalyst in the absence of PIL (EN) $\left(131 \mathrm{~m}^{2} \mathrm{~g}^{-1}\right)$; however, the biocatalysts showed reduced pore volume and diameter $\left(0.5 \mathrm{~cm}^{3} \mathrm{~g}^{-1}\right.$ and $8.2 \mathrm{~nm}(\mathrm{EN}) ; 0.4 \mathrm{~cm}^{3} \mathrm{~g}^{-1}$ and $5.9 \mathrm{~nm}$ (ENIL). For immobilized biocatalysts onto silica aerogel by physical adsorption and covalent binding in the absence of PIL has shown an increase of at least twice the pore diameter and volume compared with biocatalysts in the presence of the additive. In the absence of protic ionic liquid the biocatalysts ADS and CB had pore volume of 0.3 to $0.2 \mathrm{~cm}^{3} \mathrm{~g}^{-1}$, respectively, and in the presence of the additive the two biocatalysts ADSIL and CBIL had a pore volume of $0.6 \mathrm{~cm}^{3} \mathrm{~g}^{-1}$. Pore diameter of the immobilized biocatalysts in the absence of additive (ADS and CB) was respectively 24.36 and $17.24 \mathrm{~nm}$, and 47.48 and $56 \mathrm{~nm}$ for ADSIL and CBIL biocatalysts.

For the biocatalysts immobilized by encapsulation the use of the ionic liquid may allow penetration of the enzyme inside the bed (because the pores increase), while increasing the number of coupled enzymes and consequently the operational stability. ${ }^{37}$ Souza and colleagues ${ }^{12}$ reported that the addition of $\mathrm{C}_{5}$ during xerogel immobilization of the lipase from Burkholderia cepacia modified the porous structure of the immobilized derivatives, including enlarging the pores $(9.2 \mathrm{~nm})$ and increasing the surface area $\left(181 \mathrm{~m}^{2} \mathrm{~g}^{-1}\right)$ and pore volume $\left(0.15 \mathrm{~cm}^{3} \mathrm{~g}^{-1}\right)$. This behaviour was also reported by Zarcula and colleagues ${ }^{22}$ during encapsulation of lipase in a hydrophobic matrix with a large number of hydrophobic groups, since the hydrophobic liquids induced significant changes in the porous structure of the biocatalyst. The different results for the biocatalysts (ADS ADSIL, CB and CBIL) compared with others (EN and ENIL) can be explained by the type of immobilization; encapsulation, besides maintaining enzymes immobilized on the surface, tends to confine them in the interstices of the reticulated gel, which provides better operational stability thanks to lower enzymatic losses during the reaction or washing process in the assays of the operational stability of the immobilized biocatalysts.

\section{Thermogravimetric analysis $-T G$}

The mass losses of samples of pure silica aerogel (SA and SAIL), biocatalyst free, immobilized with additive (ENIL, CBIL and ADSIL) and without additive (EN, CB and ADS) were determined by thermogravimetric analysis (TG). The thermograms were divided into three regions to simplify discussion (Table 2). In Region I, the temperature reaches approximately $200{ }^{\circ} \mathrm{C}$, and weight loss is mainly associated with the decomposition of amino groups and extraction of the water in the surface, generally organic groups. Region II, which has temperatures between 200 and $600{ }^{\circ} \mathrm{C}$, is associated with the condensation of silanol groups and some loss of organic components $(\mathrm{C}, \mathrm{H}, \mathrm{O}$ and
$\mathrm{N}$ ) in the volatile form either present or formed until the onset of decomposition biological, including lipase and the presence of silanol groups of the unreacted TEOS, because of the incomplete sol-gel reaction, according to Mukherjee and colleagues. ${ }^{38}$ Region III, where the mass loss is associated with final dehydroxylation reactions and definitive carbonisation of organic compounds, resulting in complete degradation of the material or thermal stability, was investigated by Soares and colleagues. ${ }^{4}$

Thermogravimetric analysis showed that all the gel samples exhibit more weight loss up to $100{ }^{\circ} \mathrm{C}$, which corresponds to the evaporation of alcohol and residual water formed during the hydrolysis of $-\mathrm{Si}-\mathrm{OCH}_{2} \mathrm{CH}_{3}$ functionalities and condensation of silanol $(-\mathrm{Si}-\mathrm{OH})$ groups. ${ }^{35}$ It was observed that the SA sample showed a weight loss of approximately $17 \%$. In all cases, gels were heated up to $800{ }^{\circ} \mathrm{C}$ (data shown up to $500{ }^{\circ} \mathrm{C}$ ) and the weight loss was negligible. Therefore, thermogravimetric curves and results of loss mass of Regions I and II (Table 2) showed higher variation after the modification of silica with PIL for SAIL, ENIL and CBIL.

Table 2. Total loss of mass of the pure aerogel matrix samples, free enzyme and immobilized BC lipase in aerogel

\begin{tabular}{ccccc}
\hline Sample & $\begin{array}{c}\text { Region I } \\
\left(0-200^{\circ} \mathrm{C}\right)\end{array}$ & $\begin{array}{c}\text { Region II } \\
\left(200-600^{\circ} \mathrm{C}\right)\end{array}$ & $\begin{array}{c}\text { Region III } \\
\left(600-1000^{\circ} \mathrm{C}\right)\end{array}$ & $\begin{array}{c}\text { Total mass } \\
\text { loss }(\%)\end{array}$ \\
\hline BC Free & 8.32 & 82.32 & 9.16 & 99.81 \\
SA & 10.37 & 6.02 & 0.58 & 16.90 \\
SAIL & 41.98 & 3.67 & 0.65 & 46.31 \\
EN & 33.62 & 15.72 & 1.66 & 51.03 \\
ENIL & 16.74 & 19.40 & 1.91 & 37.99 \\
ADS & 44.73 & 2.98 & 0.71 & 48.43 \\
ADSIL & 45.62 & 3.63 & 0.89 & 50.16 \\
CB & 20.89 & 5.29 & 1.20 & 27.13 \\
CBIL & 46.63 & 3.80 & 0.98 & 51.42 \\
\hline
\end{tabular}

In the range above $750{ }^{\circ} \mathrm{C}$, either the thermal stability of the material is reached or it completely breaks down, as occurred with the sample of free biocatalyst. ${ }^{12}$ Therefore, the use of immobilized biocatalyst with higher amounts of water in the interstices of the support in Region II determined from thermogravimetric curves showed greater operational stability in the face of hydrolysis reaction for EN and ENIL and those with a larger amount of water onto surface had a better recovery of initial activity (ADS, ADSIL, CB and CBIL). In the thermogravimetric analysis the low weight loss was also observed by Souza and colleagues ${ }^{12}$ and can be attributed to the presence of un-reacted silanol groups from the TEOS, which are present in the silica because of incomplete sol-gel reactions. ${ }^{38}$ For SAIL and biocatalysts ADSIL and CBIL some of this weight loss is also owed to the removal of water molecules, which were tightly bound to the silica matrix because of the presence of the ionic liquid protic resulting from interactions between silane precursors and organic components (additives and lipase). ${ }^{12}$

\section{Fourier transform infrared spectroscopy - FTIR analysis}

The free lipase has a typical spectrum of proteins with absorption bands attached to the amide group $(\mathrm{CHONH})$ characteristic and in the band situated around $695 \mathrm{~cm}^{-1}$ (amide IV and/or V), 1600-1700 $\mathrm{cm}^{-1}$ (amide I), according to Portaccio and colleagues ${ }^{39}$ and Mohidem and Mat. ${ }^{25}$ The spectra of silica aerogels in this study and xerogel obtained by the same technique by Souza et al., ${ }^{12}$ showed similar bands at determined wavenumbers between 1000 and 1100, which 
corresponds to Si-O-R bonds of $800 \mathrm{~cm}^{-1}$ (Si-O-Si), $450 \mathrm{~cm}^{-1}$ (Si-O) and $-\mathrm{OH}$ groups in the range between 3400 and $3500 \mathrm{~cm}^{-1}$.

The spectra obtained for the immobilized biocatalysts by different immobilization methods in the absence and presence of protic ionic liquid (Figure 5) showed that beyond the characteristic bands of silanol groups there were bands in the range of wavenumbers between 1600 and $1700 \mathrm{~cm}^{-1}$ (amide I), $1390 \mathrm{~cm}^{-1}$ corresponding to $\mathrm{C}-\mathrm{H}$ groups with $\mathrm{CH}_{3}$ bonds, and $695 \mathrm{~cm}^{-1}$ (amide IV and/or V), showing the efficiency of the immobilization of $\mathrm{BC}$ lipases in the studied methods. With regard to the covalent binding method, there was a lower reduction in the band intensity characteristic Si-OR bond $\left(450 \mathrm{~cm}^{-1}\right)$.

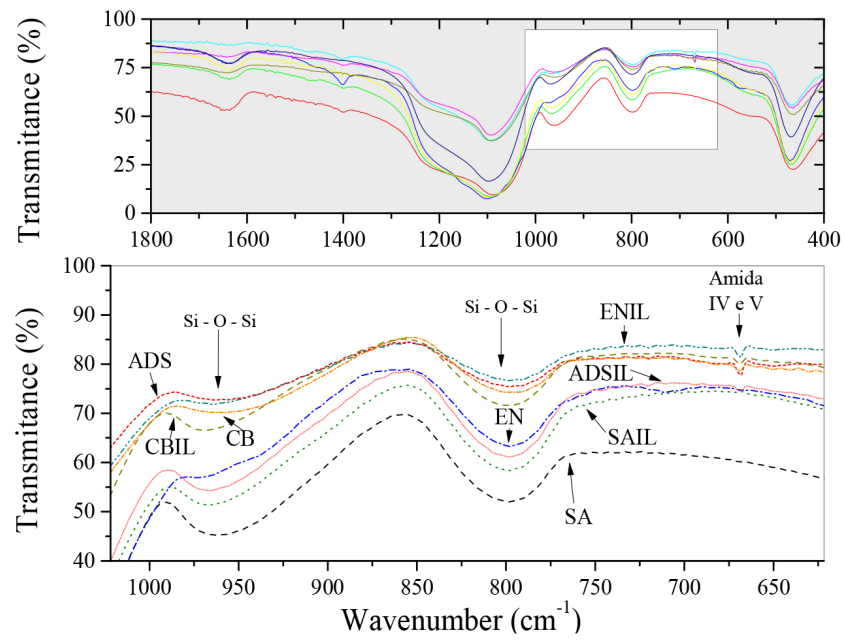

Figure 5. FTIR spectra for pure matrix and the immobilized biocatalysts from $B C$ on aerogel silica in the absence (SA, EN, $C B$ and $A D S)$ and presence of protic ionic liquid (SAIL, ENIL, CBIL and ADSIL)

The spectra (FTIR) of biocatalysts immobilized by covalent binding method showed a lower reduction in the band intensity characteristic $\mathrm{Si}-\mathrm{OR}$ bond $\left(450 \mathrm{~cm}^{-1}\right)$. This phenomenon is probably caused by the sharing of electrons because of the presence of a bifunctional agent such as glutaraldehyde spacer arm, which is capable of reacting with the $\mathrm{OH}$ groups of the support surface and the amine groups of the enzyme. ${ }^{40}$

\section{Scanning electron microscopy (SEM)}

Scanning electron micrographs of the pure silica aerogel and immobilized lipases, in the presence or absence of ionic liquid, are shown in Figure 6. The structures of the silica aerogel and immobilized biocatalyst synthesised without IL show a denser network because the shrinkage of the gel is greater during the ageing process, and exhibits a low surface porosity and irregular porous structure. On the other hand, adding a PIL additive during the synthesis of aerogel supports and the immobilization process resulted in important modifications of the general surface morphology. The SEM image shows a surface that appears to be more porous and has a more amorphous structure. This behaviour can to be attributed to the confinement of IL and also the presence of ionic liquid, which may agglomerate the silica particles. ${ }^{35}$

\section{CONCLUSION}

The BC lipase immobilized onto silica aerogel showed an increase in the total activity recovery yield of the 37 to $83 \%$, EN and ADSIL, respectively. The physical-chemical and morphological characterization justified the performance in the activity of the biocatalyst onto (a)

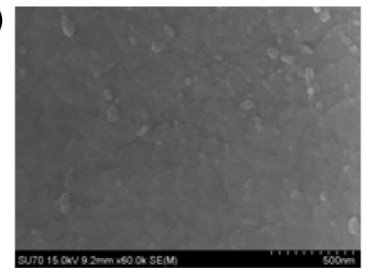

(b)

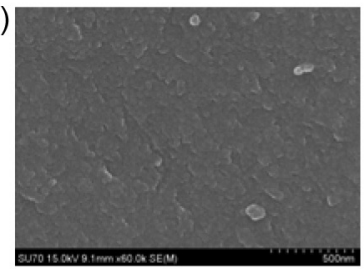

(c)

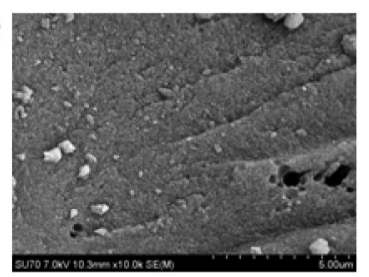

(d)

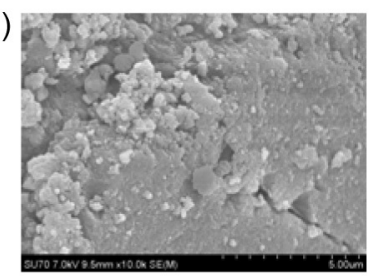

(e)

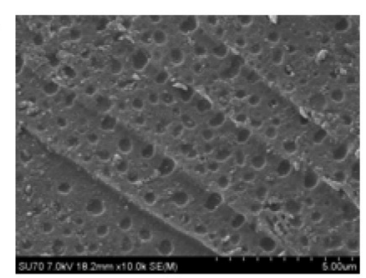

(f)

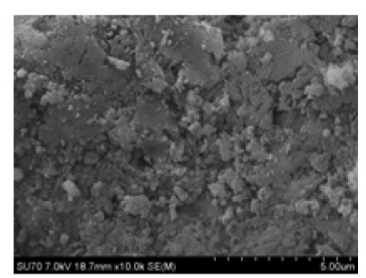

(g)

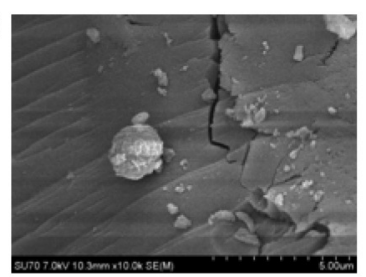

(h)

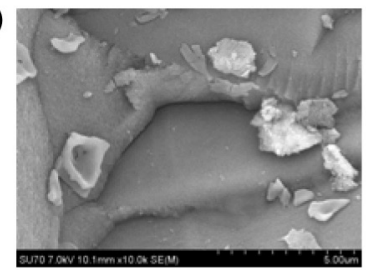

Figure 6. Scanning electron micrographs for (a) SA, (b) SAIL, (c) EN, (d) ENIL, (e) ADS, (f) ADSIL ( $g$ ) CB and (h) CBIL

novel supports. However, BC lipase immobilized into silica aerogel (encapsulation) showed the best immobilization technique and in the presence of protic ionic liquid, representing an increase in the operational stability of two for 23 reuses, ADS and ENIL, respectively.

\section{ACKNOWLEDGEMENTS}

The authors thank CNPq, CAPES, FAPITEC/SE and UNIT for their financial support of this work, and for research scholarships.

\section{REFERENCES}

1. Kistler, S. S.; Nature 1931, 127, 741.

2. Rodella, C. B.; Nunes, L. A. O.; Saeki, M. J.; Padilha, P. M.; Florentino, A. O.; Quim. Nova 2002, 25, 209.

3. Orçaire, O.; Buisson, P.; Pierre, A. C.; J. Mol. Catal. B: Enzym. 2006, 42, 106.

4. Soares, C. M. F.; Santos, O. A.; Castro, H. F.; Moraes, F. F.; Zanin, G. M.; Appl. Biochem. Biotechnol. 2004, 113, 307.

5. Novak, Z.; Habulin, M.; Krmelj, V.; Knez, Z.; J. Supercrit. Fluids 2003, 27,169 .

6. Zhang, B.; Weng, Y.; Xu, H.; Mao, Z.; Appl. Microbiol. Biotechnol. 2012, 93, 61.

7. Guisan, J. M.; Immobilization of enzymes and cell, $2^{\text {nd }}$ ed., Humana Press: New Jersey, 2006.

8. Karout, A.; Pierre, A. C.; J. Sol-Gel Sci. Technol. 2009, 52, 276.

9. Nassreddine, S.; Karout, M.; Christ, M. L.; Pierre, A. C.; J. Appl. Catal. A: Gen. 2008, 344, 70.

10. Maury, S.; Buisson, P.; Perrard, A.; Pierre, A. C.; J. Mol. Catal. B: Enzym. 2005, 32, 193. 
11. Souza, R. L.; Resende, W. C. S.; Barão, C. E.; Zanin, G. M.; Castro, H. F.; Santos, O. A. A.; Fricks, A. T.; Figueiredo, R. T.; Lima, A. S.; Soares, C. M. F.; J. Mol. Catal. B: Enzym. 2012, 84, 152.

12. Souza, R. L.; Faria, E. L. P.; Figueiredo, R. T.; Freitas, L. S.; Iglesias, M.; Mattedi, S.; Zanin, G. M.; Santos, O. A. A.; Coutinho, J. A. P.; Lima, A. S.; Soares, C. M. F.; Enzyme Microb. Technol. 2013, 52, 141.

13. Hara, P.; Hanefeld, U.; Kanerva, L. T.; Green Chem. 2009, 11, 250.

14. Karout, A.; Pierre, A. C.; J. Non-Cryst. Solid 2007, 353, 2900.

15. Lee S. H.; Doan, T. T. N.; Ha, S. H. H.; Chang, W. J.; J. Mol. Catal. B: Enzym. 2007, 47, 129.

16. Oliveira, M. V. S.; Da Rós, P. C. M.; Mattedi, S.; Castro, H. F.; Soares, C. M. F.; Lima, A. S. Acta Sci. Technol. 2014, 36, 445.

17. Alvarez, V. H.; Mattedi, S.; Martin-Pastor, M.; Aznar, M.; Iglesias, M.; J. Chem. Thermodyn. 2011, 43, 997.

18. Aslanov, L. A.; J. Mol. Liq. 2011, 162, 101.

19. Barbosa, A. S.; Silva, M. A. O.; Carvalho, N. B.; Mattedi, S.; Iglesias, M. A.; Fricks, A. T.; Lima, A. S.; Franceschi, E.; Soares, C. M. F.; Quim. Nova 2014, 37, 969.

20. Brunauer, S.; Emmett, P. H.; Teller, E.; J. Am. Chem. Soc. 1938, 60, 309.

21. Barão, C. E.; De Paris, L. D.; Dantas, J. H.; Pereira, M. M.; Filho, L. C.; Castro, H. F.; Zanin, G. M.; Moraes, F. F.; Soares, C. M. F.; Appl. Biochem. Biotechnol. 2013, 172, 263.

22. Zarcula, C.; Corîci, L.; Croitor, U. R.; Ursoiu, A.; Peter, F.; J. Mol. Catal. B: Enzym. 2010, 65, 79.

23. Moniruzzaman, M.; Nakashima, K.; Kamiya, N.; Goto, M.; Biochem. Eng. J. 2010, 48, 295.

24. Zhao, H.; J. Chem. Technol. Biotechnol. 2010, 85, 891.

25. Mohidem, N. A.; Mat, H. B.; J. Sol-Gel Sci. Technol. 2011, 61, 96.
26. Gao, S.; Wang, Y.; Wang, T.; Luo, G.; Dai, Y.; Bioresour. Technol. 2009, 100, 996.

27. Zhou, G.; Chen, Y.; Yang, S.; Microporous Mesoporous Mater. 2009, 119,223

28. Yadav, G. D.; Jadhav, S. R.; Microporous Mesoporous Mater. 2005, 86, 215.

29. Kharrat, N.; Ali, Y. B.; Marzouk, S.; Gargouri, Y-Talel.; Chaabouni, M.; Process Biochem. 2009, 46, 1083

30. Ghamgui, H.; Karra-Châabouni, M.; Gargouri, Y.; Enzyme Microb. Technol. 2004, 35, 355.

31. Karra-Châabouni, M.; Bouaziz, I.; Boufi, S.; Botelho Do Rego, A. M.; Gargouri, Y.; Colloids Surf. B 2008, 66, 168.

32. Zhou, Z.; Hartmann M.; Chem. Soc. Rev. 2013, 42, 3894.

33. Mendes, A. A.; Giordano, R. C.; Giordano, R. L. C., Castro, H. F.; J. Mol. Catal. B: Enzym. 2011, 68, 109.

34. Bai, Y-X.; Li, Y-F.; Yang, Y.; Yi, L-X.; J. Biotechnol. 2006, 125, 574.

35. Meera, K. M. S.; Sankar, R. M.; Jaisankar, S. N.; Mandal, A. B.; Colloids Surf. B 2011, 86, 292.

36. Vila-Real, H.; Alfaia, A. J.; Rosa, J. N.; Gois, P. M. P.; Rosa, M. E.; Calado, A. R. T.; J. Biotechnol. 2011, 152, 147.

37. Fernandez-Lafuente, R.; Enzyme Microb. Technol. 2009, 45, 405

38. Mukherjee, I.; Mylonakis, A.; Guo, Y.; Samuel, S. P.; Li, S.; Wei, R. Y.; Microporous Mesoporous Mater. 2009, 122, 168.

39. Portaccio, M.; Della-Ventura, B.; Mita, D. G.; Manolova, N.; Stoilova, O.; Rashkov, I.; Lepore, M.; J. Sol-Gel Sci. Technol. 2011, 57, 204.

40. Paula, A. V.; Moreira, A. B. R.; Braga, L. P.; Castro, H. F.; Bruno, L. M.; Quim. Nova 2008, 31, 35. 